TRANSACTIONS OF THE

AMERICAN MATHEMATICAL SOCIETY

Volume 280, Number 2, December 1983

\title{
A TOPOLOGICAL GROUP HAVING NO HOMEOMORPHISMS OTHER THAN TRANSLATIONS
}

BY

JAN VAN MILL

\begin{abstract}
We give an example of a (separable metric) connected and locally connected topological group, the only autohomeomorphisms of which are group translations.
\end{abstract}

1. Introduction. The aim of this paper is to present an example of a connected and locally connected topological group $G$ having no homeomorphisms other than group translations. It turns out that $G$ is uniquely homogeneous, i.e. for any pair of points $x$, $y \in G$ there is a unique homeomorphism taking $x$ onto $y$. Our results were motivated, on the one hand, by the result due to Barit and Renaud [2] that a uniquely homogeneous space cannot be compact, or locally compact and locally connected, and, on the other hand, by the recent result due to Dobrowolski and Torunczyk [5] that a topologically complete ANR group is either a Lie group or a Hilbert space manifold and consequently these groups have many homeomorphisms other than those derived from their underlying algebraic structure.

Our example answers many natural questions in the negative. To my knowledge, it is the first example of a uniquely homogeneous space. In addition, it is an example of a homogeneous topological space the autohomeomorphism group of which is Abelian. We will derive many other strange properties of $G$. For example, if $x \in G$ then the space $G \backslash\{x\}$ is rigid, i.e. has no autohomeomorphisms other than the identity.

We use a method originally due to Kuratowski [14] which was later rediscovered or used by many authors, see e.g. $[6,7,9,10,16-20]$. Some specific ideas needed to apply Kuratowski's technique in the present construction are implicit in [16-18].

2. Preliminaries. Space means separable metric topological space. A space is called topologically complete provided that there is a complete metric on the space that generates the topology. The following classical results will be important in our construction.

2.1. Lemma. (a) (Lavrentieff [15]) Let $X$ be a space and let $Y$ be topologically complete. If $A \subset X$ and if $f: A \rightarrow Y$ is continuous, then there is a $G_{\delta}$-subset $A^{\prime} \subset X$ such that $f$ can be extended to a map $\bar{f}: A^{\prime} \rightarrow Y$.

Received by the editors February 8, 1982.

1980 Mathematics Subject Classification. Primary 22A05, 54G20.

Key words and phrases. Topological group, homeomorphism, unique homogeneity.

(C)1983 American Mathematical Society $0002-9947 / 83 \$ 1.00+\$ .25$ per page 
(b) (Sierpinski [21]) If $X$ is a continuum and if $Q$ is a partition of $X$ in countably many closed sets, then at most one element of $Q$ is nonempty.

(c) (Hausdorff [13]) If X is topologically complete and uncountable, then $X$ contains a Cantor set. A topologically complete space is therefore either countable or has cardinality continuum.

A cardinal is an initial ordinal and an ordinal is the set of smaller ordinals. $c$ denotes $2^{\aleph_{0}}$.

The domain and range of a function $f$ will be denoted by $\operatorname{dom}(f)$ and $\operatorname{range}(f)$, respectively. Henceforth, all functions will be continuous. Observe that the cardinality of the collection of all $G_{\delta}$-subsets of a given space is at most c. This implies that if $X$ and $Y$ are spaces, then the collection

$$
\mathcal{F}=\left\{f: \operatorname{dom}(f) \text { is a } G_{\delta} \text {-subset of } X \text { and } \operatorname{range}(f) \subset Y\right\}
$$

has cardinality at most $\mathrm{c}$.

If $G$ is a group and if $A \subset G$ then $\langle A\rangle$ denotes the subgroup of $G$ generated by $A$. A group is called Boolean provided that each element of the group has order at most 2. Observe that a Boolean group is Abelian. If $G$ is an Abelian group then we let " +" denote the operation on $G$. Let $G$ be a Boolean group and let $H \subset G$ be a subgroup. It is easy to verify that for each $x \in G$ the following formula holds:

$$
\langle H \cup\{x\}\rangle=H \cup(x+H) \text {. }
$$

If $A$ and $B$ are sets, then $A \triangle B$ denotes their symmetric difference, i.e. $A \triangle B=$ $(A \backslash B) \cup(B \backslash A)$. As usual, $\lambda$ denotes Lebesgue measure on $[0,1]$. Let $\Re$ denote the family of all Lebesgue measurable subsets of $[0,1]$ and Let $\Re$ be the ideal of null-sets. The quotient algebra $\Re / \Re$ will be denoted by $\mathcal{G}$. If $A \in \Re$ then $[A]$ denotes the $\mathcal{G}$-equivalence class of $A$. Metrize $\mathcal{G}$ by $d([A],[B])=\lambda(A \triangle B)$. As is well known, $d$ is a complete metric on $\mathcal{G}$ [12, Exercise 40.1] which, in addition, is convex [12, Exercise 40.8]. This easily implies that $\mathcal{G}$ is path-connected. Since obviously $\mathcal{G} \times \mathcal{G} \approx \mathcal{G}$, we conclude that for any pair of points $x, y \in \mathcal{G}$ there is a disc $D \subset \mathcal{G}$, i.e. a topological copy of $[0,1] \times[0,1]$, containing both $x$ and $y$. This will be of crucial importance later on. Bessaga and Pelczyński [3, VI, 7.2] have shown that $\mathcal{G}$ is homeomorphic to the separable Hilbert space $l_{2}$, so using their result the above property of $\mathcal{G}$ becomes a triviality. If we define an operation $+: \mathcal{G} \times \mathcal{G} \rightarrow \mathcal{G}$ by $[A]+[B]=[A \triangle B]$, then $\mathcal{G}$ with this operation becomes a Boolean topological group [12, 167]. We have given a well-known example of a topological group $G$ satisfying the following properties:

(1) $G$ is topologically complete.

(2) for all $x, y \in G$ there is a disc $D \subset G$ with $x, y \in D$.

(3) $G$ is Boolean.

3. The construction. We will now present the main result in this paper.

3.1. ThEOREM. Let $G$ be a topological group which is topologically complete and Boolean and which, moreover, has the property that any pair of points $x, y \in G$ is contained in a disc $D \subset G$. Then $G$ contains a dense connected subgroup $H$ such that 
each autohomeomorphism of $H$ is a translation. Moreover, if $G$ has the property that for all open $U \subset G$ and $x \in U$, there is an open $V \subset U$ with $x \in V \subset U$ such that any pair of points of $V$ is contained in a disc $D \subset U$, then $H$ is locally connected.

Since the proof of this result is rather lengthy, we break it up in several pieces.

3.2. Construction of $H$. Let $\mathscr{F}=\left\{f: \operatorname{dom}(f)\right.$ is a $G_{\delta}$-subset of $G$ and $\left.\operatorname{range}(f) \subset G\right\}$. Since, as was observed in $\S 2,|\mathscr{F}| \leqslant c$, we can enumerate $\mathscr{F}$ by $\left\{f_{\alpha}: \alpha<c, \alpha\right.$ even $\}$. We choose the enumeration such that each $f \in \mathscr{F}$ is listed $c$ times. It is clear that this is possible. Let $\left\{K_{\alpha}: \alpha<\mathfrak{c}, \alpha\right.$ odd $\}$ enumerate all Cantor sets in $G$. By transfinite induction, for every $\alpha<\mathrm{c}$ we will construct subgroups $H_{\alpha} \subset G$ and subsets $V_{\alpha} \subset G$ such that

(1) if $\beta<\alpha$ then $H_{\beta} \subset H_{\alpha}, V_{\beta} \subset V_{\alpha}$, and $H_{\alpha} \cap V_{\alpha}=\varnothing$,

(2) $\left|H_{\alpha}\right| \leqslant|\alpha| \cdot \boldsymbol{\aleph}_{0}$ and $\left|V_{\alpha}\right| \leqslant|\alpha| \cdot \boldsymbol{\aleph}_{0}$,

(3) if $\alpha$ is odd then $H_{\alpha} \cap K_{\alpha} \neq \varnothing$,

(4) if $\alpha$ is even and if $\left|\left\{x \in \operatorname{dom}\left(f_{\alpha}\right): f_{\alpha}(x) \notin\left\langle\cup_{\beta<\alpha} H_{\beta} \cup\{x\}\right\rangle\right\}\right|=\mathfrak{c}$, then there is a point $x \in \operatorname{dom}\left(f_{\alpha}\right) \cap\left(H_{\alpha} \backslash \cup_{\beta<\alpha} H_{\beta}\right)$ such that $f_{\alpha}(x) \in V_{\alpha}$.

Suppose that we have completed the construction for all $\beta<\alpha$, where $\alpha<\mathrm{c}$.

Case 1. $\alpha$ is odd.

For convenience, put $H^{\alpha}=\cup_{\beta<\alpha} H_{\beta}$ and $V^{\alpha}=\cup_{\beta<\alpha} V_{\beta}$. Observe that $\left|H^{\alpha}\right| \leqslant$ $|\alpha| \cdot \boldsymbol{\aleph}_{0}<\mathrm{c}$ and, similarly, that $\left|V^{\alpha}\right| \leqslant|\alpha| \cdot \boldsymbol{\aleph}_{0}<\mathrm{c}$. Since $\left|K_{\alpha}\right|=\mathfrak{c}$, we can pick a point

$$
x \in K_{\alpha} \backslash\left(H^{\alpha}+V^{\alpha}\right) .
$$

Define $H_{\alpha}=\left\langle H^{\alpha} \cup\{x\}\right\rangle$ and $V_{\alpha}=V^{\alpha}$. An easy check shows that $H_{\alpha}$ and $V_{\alpha}$ are as required.

Case 2. $\alpha$ is even and if $S=\left\{x \in \operatorname{dom}\left(f_{\alpha}\right): f_{\alpha}(x) \notin\left\langle H^{\alpha} \cup\{x\}\right\rangle\right\}$ then $|S|<c$.

Define $H_{\alpha}=H^{\alpha}$ and $V_{\alpha}=V^{\alpha}$.

Case 3. $\alpha$ is even and $|S|=$ c.

By the same argument as in Case 1, we can find a point

$$
x \in S \backslash\left(\left(H^{\alpha}+V^{\alpha}\right) \cup H^{\alpha}\right) .
$$

Define $H_{\alpha}=\left\langle H^{\alpha} \cup\{x\}\right\rangle$ and $V_{\alpha}=V^{\alpha} \cup\left\{f_{\alpha}(x)\right\}$. Since

$$
H_{\alpha} \cap V_{\alpha}=\left(H^{\alpha} \cup\left(x+H^{\alpha}\right)\right) \cap\left(V^{\alpha} \cup\left\{f_{\alpha}(x)\right\}\right)=\varnothing,
$$

we see that $H_{\alpha}$ and $V_{\alpha}$ are as required.

Now put $H=\cup_{\alpha<c} H_{\alpha}$. We claim that $H$ is as required. For later use we prove a statement which is slightly more general than the statement we claimed to be true for $H$.

3.3. Let $A \subset H$ be countable and let $f: H \backslash A \rightarrow H$ be a map with the property that each point-inverse is at most countable. Then there is a translation $h: G \rightarrow G$ with $h \mid H \backslash A=f$.

By Lemma 2.1(a), let $S$ be a $G_{\delta}$-subset of $G$ such that $f$ can be extended to a map $\bar{f}: S \rightarrow H$. Observe that $\left\{\alpha<c: \bar{f}=f_{\alpha}\right\}$ has cardinality c.

Case 1. For each $\alpha$ such that $\bar{f}=f_{\alpha},\left|\left\{x \in S: f_{\alpha}(x) \notin\left\langle\cup_{\beta<\alpha} H_{\beta} \cup\{x\}\right\rangle\right\}\right|=\mathrm{c}$. Then by (4), there is a set $B \subset \operatorname{dom}(\bar{f}) \cap H$ of cardinality c such that $f(B) \cap H=\varnothing$. 
Take a point $x \in B \backslash A$. Then $x \in H \backslash A$ and since $\bar{f}$ extends $f$, we conclude that $f(x) \notin H$, which is a contradiction.

Case 2. Not Case 1. Take $\alpha<\mathrm{c}$ such that $\bar{f}=f_{\alpha}$ and

$$
\left|\left\{x \in S: f_{\alpha}(x) \notin\left\langle\bigcup_{\beta<\alpha} H_{\beta} \cup\{x\}\right\rangle\right\}\right|<c .
$$

Let $H^{\alpha}=\cup_{\beta<\alpha} H_{\beta}$ and $T=\left\{x \in S: f_{\alpha}(x) \notin\left(H^{\alpha}+\left(x+H^{\alpha}\right)\right)\right\}$. By assumption, $|T|<c$. Take $h \in H^{\alpha}$ and assume that $f_{\alpha}^{-1}(h)$ is uncountable. Since $f_{\alpha}^{-1}(h)$ is a closed subspace - of the space $S$, which is a $G_{\delta}$-subset of $G, f_{\alpha}^{-1}(h)$ contains a family of $c$ pairwise disjoint Cantor sets (Lemma 2.1(c)). From (3) we conclude that $\left|f_{\alpha}^{-1}(h) \cap H\right|=\mathfrak{c}$, whence $\left|f_{\alpha}^{-1}(h) \cap(H \backslash A)\right|=c$. Since $f_{\alpha}$ extends $f$, this contradicts our assumptions on $f$. We conclude that

$$
\left|f_{\alpha}^{-1}\left(H^{\alpha}\right)\right| \leqslant\left|H^{\alpha}\right| \cdot \boldsymbol{\aleph}_{0} \leqslant|\alpha| \cdot \boldsymbol{\aleph}_{0} \cdot \boldsymbol{\aleph}_{0}<\mathfrak{c} .
$$

For each $h \in H^{\alpha}$ define

$$
E_{h}=\left\{x \in S: f_{\alpha}(x)=x+h\right\} .
$$

Observe that each $E_{h}$ is closed in $S$ and that the collection $\left\{E_{h}: h \in H^{\alpha}\right\}$ is pairwise disjoint. If $h \in H^{\alpha}$ define $F_{h}=E_{h} \backslash f_{\alpha}^{-1}\left(H^{\alpha}\right)$. We claim that at most one of the collection $\left\{F_{h}: h \in H^{\alpha}\right\}$ is nonempty. Suppose, to the contrary, that there exist distinct points $s, t \in H^{\alpha}$ such that $F_{s} \neq \varnothing$ and $F_{t} \neq \varnothing$. Pick points $x \in F_{s}$ and $y \in F_{t}$. By assumption, there is a disc $D \subset G$ containing both $x$ and $y$. Since $S$ is a $G_{\delta}$, the complement $G \backslash S$ of $S$ is the union of countably many closed subsets of $G$, none of which can contain a Cantor set by (3). We conclude that $G \backslash S$ is countable, Lemma 2.1(c). Therefore, $\left|f_{\alpha}^{-1}\left(H^{\alpha}\right) \cup(G \backslash S)\right|<c$ which implies that we can find an $\operatorname{arc} J \subset D$ connecting $x$ and $y$ such that

$$
J \cap\left(f_{\alpha}^{-1}\left(H^{\alpha}\right) \cup(G \backslash S) \cup T\right)=\varnothing,
$$

whence

$$
J \subset \bigcup_{h \in H^{\alpha}} F_{h} \subset \bigcup_{h \in H^{\alpha}} E_{h} .
$$

Put $K=\left\{h \in H^{\alpha}: F_{h} \cap J \neq \varnothing\right\}$. By assumption, $|K| \geqslant 2$, whence, by Sierpiński's Lemma (Lemma 2.1(b)), $|K|>\boldsymbol{\aleph}_{0}$. Also, $|K| \leqslant\left|H^{\alpha}\right|<\mathfrak{c}$ (so in case the Continuum Hypothesis holds, at this stage of the proof we have already derived a contradiction). We conclude that $K$ cannot be topologically complete, Lemma 2.1(c), and that, in particular, $K$ is not closed in $G$. Let $k_{n} \in K(n=1,2, \ldots)$ be a sequence of points of $K$ converging to a point $k \notin K$. For each $n$, take a point $x_{n} \in J \cap F_{k_{n}}$. By compactness of $J$, we may assume that $\lim _{n \rightarrow \infty} x_{n}=x \in J$. Then

$$
f_{\alpha}(x)=\lim _{n \rightarrow \infty} f_{\alpha}\left(x_{n}\right)=\lim _{n \rightarrow \infty}\left(x_{n}+k_{n}\right)=x+k,
$$

since $\lim _{n \rightarrow \infty} x_{n}=x, \lim _{n \rightarrow \infty} k_{n}=k$ and " + " is continuous. Since $x \in J$, there is an $h \in K$ such that $x \in F_{h} \subset E_{h}$. Consequently, $f_{\alpha}(x)=x+h$, which implies that $k=h \in K$, which is a contradiction. 
We conclude that at most one of the collection $\left\{F_{h}: h \in H^{\alpha}\right\}$ is nonempty. If each $F_{h}$ is empty, then $f_{\alpha}(H \backslash T) \subset H^{\alpha}$ and consequently, $|H|<\mathfrak{c}$, by (*), which obviously contradicts (3). Therefore, there is a unique point $h \in H^{\alpha}$ such that $F_{h} \neq \varnothing$. Suppose that $E_{h} \neq S$. Then $S \backslash E_{h}$ is nonempty and open in $S$. Since $|G \backslash S| \leqslant \boldsymbol{\aleph}_{0}$ and since each nonempty open subspace of $G$ has cardinality c ( $G$ is path-connected), this implies that $\left|S \backslash E_{h}\right|=\mathfrak{c}$, which contradicts (*). Consequently, $E_{h}=S$ which completes the proof of 3.3 .

3.4. $H$ is connected. Moreover, if $G$ has the property that for all open $U \subset G$ and $x \in U$, there is an open $V \subset U$ with $x \in V \subset U$ such that any pair of points of $V$ is contained in a disc $D \subset U$, then $H$ is locally connected.

Suppose, to the contrary, that $H$ is not connected, i.e. $H=U \cup V$, where $U$ and $V$ are disjoint nonempty open subsets of $H$. Since $H$ is dense in $G$, we can find disjoint open sets $U^{\prime}, V^{\prime} \subset G$ with $U^{\prime} \cap G=U$ and $V^{\prime} \cap H=V$. Then $K=G \backslash\left(U^{\prime} \cup V^{\prime}\right)$ is closed and therefore has to be countable since it misses $H$ ( $H$ intersects every Cantor set in $G$ ). Take $x \in U^{\prime}$ and $y \in V^{\prime}$. By assumption, there is a disc $D \subset G$ containing both $x$ and $y$. Then $K \cap D$ separates $D$ and since no countable set separates $D$, this is a contradiction.

To prove that $H$ is locally connected under the given hypotheses, first observe that $G$ is locally connected. We will show that if $W \subset G$ is open and connected, then $W \cap H$ is connected, which proves, among others, that $H$ is locally connected. Let $W \subset G$ be open and connected, and suppose that $W \cap H$ is not connected, i.e. $W \cap H=W_{0} \cup W_{1}$, where $W_{0}$ and $W_{1}$ are disjoint nonempty open subsets of $H$. As above, find disjoint open subsets $W_{0}^{\prime}, W_{1}^{\prime} \subset W$ such that $W_{i}^{\prime} \cap H=W_{i}(i=0,1)$. Put $L=W \backslash\left(W_{0}^{\prime} \cup W_{1}^{\prime}\right)$. As above, $L$ is countable. Since $H \cap W$ is dense in $W$, and since $W$ is connected, $L$ is nonempty. In fact, there is even a point $x \in L$ which is a limit point both from $W_{0}^{\prime}$ and $W_{1}^{\prime}$. By assumption, there is a neighborhood $V$ of $x$ which is contained in $W$ such that any pair of points of $V$ is contained in a disc $D \subset W$. Since $V$ intersects both $W_{0}^{\prime}$ and $W_{1}^{\prime}$, we may, therefore, conclude that there exists a disc $D \subset W$ such that $D$ intersects both $W_{0}^{\prime}$ and $W_{1}^{\prime}$. Since $L$ is countable, this is a contradiction.

This completes the proof of the theorem.

3.5. REMARK. From now on, $H$ will denote the subgroup of $\mathcal{G}$ that we get from the proof of Theorem 3.1. Since $\mathcal{G} \approx l_{2}$, or by a direct argument, $\mathcal{G}$ satisfies all conditions required in Theorem 3.1. We therefore conclude that $H$ is a Boolean group which is connected and locally connected and which has the property that all autohomeomorphisms are translations.

3.6. REMARK. In fact, the assumption on $G$ that any pair of points is contained in a disc $D \subset G$ can be weakened considerably. The reader can easily check that the only thing we need for the proof, is the following:

for all $x, y \in G$ there is a family $\mathcal{K}$ of $c$ subcontinua of $G$ each containing $x$ and $y$ such that if $K_{0}$ and $K_{1} \in \mathscr{K}$ are distinct, then

$$
\left(K_{0} \cap K_{1}\right) \backslash\{x, y\}=\varnothing .
$$


4. A result under the Continuum Hypothesis. We have seen that there are topological groups having no homeomorphisms other than translations. Under the Continuum Hypothesis (abbreviated $\mathrm{CH}$ ) we can do better. The groups we constructed then have the property that every self-map is either constant or a translation. We will sketch the proof. Our notation and terminology is the same as in the proof of Theorem 3.1. Let $f: H \rightarrow H$ be a self-map, and let $S$ be a $G_{\delta}$-subset of $G$ such that $f$ can be extended to a map $\bar{f}: S \rightarrow \mathcal{G}$. Fix $\alpha<$ c such that $\bar{f}=f_{\alpha}$. Just as in the proof of Theorem 3.1, we find that if $T=\left\{x \in S: f_{\alpha}(x) \notin\left(H^{\alpha} \cup\left(x+H^{\alpha}\right)\right)\right\}$ then $|T|<\mathfrak{c}$, whence by $\mathrm{CH},|T| \leqslant \boldsymbol{\aleph}_{0}$. For each $h \in H^{\alpha}$ define

$$
A_{h}=\left\{x \in S: f_{\alpha}(x)=h\right\} \quad \text { and } \quad B_{h}=\left\{x \in S: f_{\alpha}(x)=x+h\right\} \text {, }
$$

respectively. Observe that if $h, g \in H^{\alpha}$ are distinct then

$$
\begin{gathered}
A_{h} \cap A_{g}=\varnothing, \quad B_{h} \cap B_{g}=\varnothing \text { and } \\
A_{h} \cap B_{g} \subset H^{\alpha} .
\end{gathered}
$$

If $h \in H^{\alpha}$, define $A_{h}^{\prime}=A_{h} \backslash H^{\alpha}$ and $B_{h}^{\prime}=B_{h} \backslash H^{\alpha}$. Then the collection

$$
\circlearrowright=\left\{A_{h}^{\prime}: h \in H^{\alpha}\right\} \cup\left\{B_{h}^{\prime}: h \in H^{\alpha}\right\}
$$

is pairwise disjoint and

$$
\left|G \backslash \bigcup_{h \in H^{\alpha}}\left(A_{h}^{\prime} \cup B_{h}^{\prime}\right)\right| \leqslant|G \backslash S|+|T|+\left|H^{\alpha}\right| \leqslant \boldsymbol{\aleph}_{0} .
$$

Consequently, by using Sierpiński's Lemma 2.1(b), we can conclude by precisely the same construction as in the proof of Theorem 3.1 that exactly one of the collection $C$ is nonempty. If $A_{h}^{\prime} \neq 0$, then $f$ is constant and if $B_{h}^{\prime} \neq \varnothing$ then $f$ is a translation.

We have completed the proof of the following

4.1. THEOREM (CH). There is a connected and locally connected topological group $H$ such that each self-map $f: H \rightarrow H$ (not necessarily onto) is either constant or a translation.

We do not know whether the above theorem is true without the assumption of the $\mathrm{CH}$. Notice that for the case of homeomorphisms we found a trick which was used to avoid the $\mathrm{CH}$. We have tried for some time to find a similar trick for self-maps, but although we believe that there should be one handling this more general case, we did not succeed.

4.2. Question. Does there exist a homogeneous continuum $X$ such that each self-map $f: X \rightarrow X$ is either constant or a homeomorphism?

5. Unique homogeneity. Burgess [4] asked whether uniquely homogeneous continua exist and Ungar [22], using deep results of Effros [8], showed that if $X$ is uniquely homogeneous and either compact or locally compact and locally connected, then $X$ is an Abelian topological group, and the group of topological automorphisms of $X$ is trivial. Barit and Renaud [2] in their paper, There are no uniquely homogeneous spaces, show that if $G$ is a locally compact topological group with $\operatorname{Aut}(G)=$ \{id $\}$, then $G=\{0\}$ or $G=Z_{2}$, thus answering Burgess' question in the negative. Since the examples we get from Theorem 3.1 are Boolean groups, it easily follows 
that they are uniquely homogeneous. This shows that the title of the Barit and Renaud paper is somewhat misleading. In addition, it also shows that in their result the local compactness is essential, since our group $H$ has no nontrivial topological automorphisms. In addition, since $H$ intersects all Cantor sets in $\mathcal{G}$, it easily follows that $H$ is Baire. We therefore conclude that on the one hand, conpact or locally compact and locally connected uniquely homogeneous spaces do not exist, but on the other hand, Baire uniquely homogeneous spaces do exist. This suggests the following question.

5.1. Question. Does there exist a uniquely homogeneous topologicaily complete space (containing more than one point $)$ ?

All examples we have of uniquely homogeneous spaces are topological groups. The way we constricted these examples shows that we cannot avoid this. This suggests the following question.

5.2. Question. Does there exist a uniquely homogeneous space that does not admit the structure of a topological group?

6. Rigid subspaces. We will proceed to show that $H$ has a very curious additional property.

6.1. TheOrem. If $x \in H$, then $H \backslash\{x\}$ is rigid.

Proof. Let $h: H \backslash\{x\} \rightarrow H \backslash\{x\}$ be a homemorphism. By 3.3, there is a translation $g: \mathcal{G} \rightarrow \mathcal{G}$ such that $g \mid H \backslash\{x\}=h$. Assume that $g(y)=y+a$ for all $y \in \mathcal{G}$. If $a$ is not the identity, then $x+a \neq x$ : consequently, $x+a \in H \backslash\{x\}$. Therefore,

$$
h(x+a)=g(x+a)=x+a+a=x,
$$

which is a contradiction of course. So $a$ must be $t^{\prime} \mathrm{e}$ identity of $\mathcal{G}$, whence $h$ is the identity homeomorphism of $H \backslash\{x\}$.

The above result shows that there are rigid spaces that can be made into a topological group by adding just one point.

The reader is encouraged to check that under $\mathrm{CH}$, the space $H \backslash\{x\}$ has the property that any self-map is either constant or the identity. Again, we do not know whether $\mathrm{CH}$ is essential here.

The reader can easily cook up many more improbable properties of $H$ in the same spirit.

7. Remarks. R. D. Anderson [1] showed that the autohomeomorphism group Aut $h(X)$ of many topological spaces $X$ is algebraically simple. The easiest examples of spaces $X$ for which $\operatorname{Aut} h(X)$ is not simple are spaces for which Auth $(X)$ is Abelian. There are such spaces, since de Groot and Wille [11] showed that each countable group is isomorphic to the autohomeomorphism group of some 1-dimensional Peano continuum: see also de Groot [10]. While discussing this with Eric van Douwen, we observed that we did not know of an example of a homogeneous space the autohomeomorphism group of which is Abelian. Our example $H$ is isomorphic to Aut $h(H)$ and consequently Aut $h(H)$ is Abelian.

REMARK (added in February 1983). In a forthcoming paper entitled $A$ uniquely homogeneous space need not be a topological group (to appear in Fund. Math.), I will answer Question 5.2. 


\section{REFERENCES}

1. R. D. Anderson, The algebraic simplicity of certain groups of homeomorphisms, Amer. J. Math. 80 (1958), 955-963.

2. W. Barit and P. Renaud, There are no uniquely homogeneous spaces, Proc. Amer. Math. Soc. 68 (1978), 385-386.

3. C. Bessaga and A. Pelczyński, Selected topics in infinite-dimensional topology, PWN, Warsaw, 1975.

4. C. E. Burgess, Homogeneous continua, Summary of Lectures and Seminars, Summer Institute on Set Theoretic Topology, Univ. Wisconsin, 1955, pp. 75-78.

5. T. Dobrowolski and H. Torunczyk, Separable complete ANR's admitting a group structure are Hilbert manifolds, Topology Appl. 21 (1981), 229-235.

6. E. K. van Douwen, A compact space with a measure that knows which sets are homeomorphic, Adv. Math. (to appear).

7. B. Dushnik and E. W. Miller, Concerning similarity transformations of linearly ordered sets, Bull. Amer. Math. Soc. 46 (1940), 322-326.

8. E. G. Effros, Transformation groups and C-algebras, Ann. of Math. (2) 81 (1965), 38-55.

9. A. Glass, Y. Gurevich, W. Holland and S. Shelah, Rigid homogeneous chains, Math. Proc. Cambridge Philos. Soc. 89 (1981), 7-17.

10. J. de Groot, Groups represented by homeomorphism groups, Math. Ann. 138 (1959), 80- 102.

11. J. de Groot and R. J. Wille, Rigid continua and topological group-pictures, Arch. Math. (Basel) 9 (1958), 441-446.

12. P. R. Halmos, Measure theory, Van Nostrand, Princeton, N.J., 1950.

13. F. Hausdorff, Grundzüge der Mengenlehre, Leipzig, 1914.

14. K. Kuratowski, Sur la puissance de l'ensemble des "nombres de dimension" de M. Fréchet, Fund. Math. 34 (1925), 201-208.

15. M. Lavrentieff, Contribution à la théorie des ensembles homéomorphes, Fund. Math. 8 (1925), 201-208.

16. J. van Mill, Strong local homogeneity does not imply countable dense homogeneity, Proc. Amer. Math. Soc. 84 (1982), 143-148.

17. Homogeneous subsets of the real line which do not admit the structure of a topological group, Indag. Math. 44 (1982), 37-43.

18. _ Representing countable groups by homeomorphism groups in Hilbert space, Math. Ann. 259 (1982), 321-329.

19. T. Ohkuma, Sur quelques ensembles ordonnés linéairement, Fund. Math. 43 (1955), 326-337.

20. J. Roitman, The measure of rigidly homogeneous chains (to appear).

21. W. Sierpiński, Un théorème sur les continus, Tôhoku Math. J. (2) 13 (1918), 300-303.

22. G. Ungar, On all kinds of homogeneous spaces, Trans. Amer. Math. Soc. 212 (1975), 393-400.

Subfaculteit Wiskunde, Vrije Universiteit, De Boelelaan 1081, Amsterdam, 1081 HV The NETHERLANDS 